\title{
Redefining Simulation Fidelity for Healthcare Education
}

\section{Jimmy Kyaw Tun (Corresponding Author)}

E-mail: jimms@doctors.net.uk

Affiliation: Barts Health NHS Trust, UK

Address: The Royal London Hospital, Whitechapel, London, E11BB, UK

Tel: $+44(0) 7817578790$

\section{Guillaume Alinier}

E-mail: g.alinier@herts.ac.uk

Affiliation: University of Hertfordshire, UK, and Hamad Medical Corporation Ambulance Service, Qatar

Address: School of Health and Social Work, University of Hertfordshire, College Lane, Hatfield, AL10 9AB, UK

Tel: +974 33512900

Tel: $+44(0) 7960934643$

\section{Jessica Tang}

E-mail: tjj571@ha.org.hk

Affiliation: Hospital Authority, Hong Kong

Address: 147B Argyle Street, Kowloon, Hong Kong

Tel: +85223007184

Fax: +85228815848

\section{Roger L. Kneebone}

E-mail: r.kneebone@imperial.ac.uk

Affiliation: Division of Surgery, Department of Surgery and Cancer, Faculty of Medicine, Imperial College London, UK

Address: Clinical Skills Centre, 2nd Floor Paterson Building, St Mary's Hospital, Praed Street, London, W2 1NY, UK 
Tel: $+44(0) 2033127930$

Tel: +44 (0)7977909707

Fax: $+44(0) 2033121810$

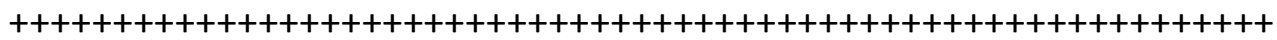

Jimmy Kyaw Tun

Imperial College, United Kingdom

Guillaume Alinier

University of Hertfordshire, UK

Hamad Medical Corporation Ambulance Service, Qatar

Jessica Tang

Hospital Authority, Hong Kong

Roger L. Kneebone

Imperial College, United Kingdom

\section{Abstract}

Background. "Fidelity" - an intrinsic property of simulation is crucial to simulation design and educational effectiveness. Yet the term fidelity is inconsistently used, which makes it difficult to draw inferences from current literature and translate research to practice.

Aim. In this article, we attempt to bring some clarity to the term simulation fidelity in healthcare education.

Method. We argue against the notion that high-fidelity simulation requires complete and faithful replication of reality, but instead the accurate representation 
of real world cues and stimuli. We address a number of issues surrounding the term fidelity and how it is currently used in the literature.

Result. In recognising the limitations of current methods of describing fidelity in the literature, we propose an alternative 3-dimensional framework for fidelity along the axes of the patient, clinical scenario, and healthcare facilities as a means for more precise and practical positioning of current healthcare simulation activities.

Conclusion. All aspects of fidelity significantly hinge on the learners' perceived realism of the context of the learning episode as opposed to any one particular element such as the technology used.

KEYWORDS: Clinical facilities; Clinical scenario; Cues; Fidelity; Deception; Educational effectiveness; Environment; Fidelity; Framework; Healthcare; Healthcare simulation; Learner perception; Learning experience; Patient; Realism; Representation; Scenario design; Simulation-based training; Simulation design

Simulation-based education is increasingly used in healthcare for training, research, and assessment purposes in response to the challenges of modern healthcare education and patient safety (Alinier \& Platt, 2014; Kneebone et al., 2009; Ziv et al., 2003). Despite its promises, more evidence of when and how simulation should be used is needed, resulting in an increase in healthcare simulation-related scientific literature (McGaghie et al., 2010). However, this literature can be difficult to interpret and translate to other contexts, in part due to the varied interpretations and applications of terminology. In particular, term fidelity is often inconsistently used in the literature (Paige \& Morin, 2013). Yet fidelity has been long considered to be a crucial factor in terms of the design, cost, and educational effectiveness of a simulation (AGARD, 1980; Beaubien \& Baker, 2004; Gerathewohl, 1969). This 
terminological confusion presents a number of problems to educators and researchers in healthcare simulation (Alessi, 2002; Chiniara et al., 2013) and encouraged the publication of standards in an attempt to clarify this issue (Meakim et al., 2013). On the industry side, the same issue prevails among manufacturers within their marketing literature. This creates a problem of parity when comparing various products blindly looking at their descriptors instead of understanding the actual functionality and features of their products in terms of so called fidelity. Without a clear concept of what fidelity is, it is difficult to design simulations to a required level of fidelity to promote transfer of learning.

It is also difficult to determine what level of fidelity is required for educational effectiveness. For instance, it is generally acknowledged that simulations of different fidelity have varying educational value for different learners and different learning objectives (Bredmose et al., 2010; Lapkin \& Levett-Jones, 2011; Lee et al., 2008; Levett-Jones et al., 2011; Norman et al., 2012), and theoretical models have been proposed (Alinier, 2007; Chiniara et al., 2013). Yet despite the research available, generalisable theories that are firmly based on empirical evidence, which can guide fidelity requirements for education are lacking (Aggarwal et al., 2010). Recently, research comparing high versus low-fidelity simulations are unequivocal, some supporting higher fidelity, whilst others supporting lower (Borodzicz, 2004; Bredmose et al., 2010; Dieckmann et al., 2007a; Kardong-Edgren et al., 2007, Lapkin and Levett-Jones, 2011).

A key reason for the terminological issues is the range of definitions used in the current literature (Pace, 1998). In the early 1990s Lane \& Alluisi (1992) identified over 22 different definitions of simulation fidelity - a figure that is likely to have increased since. Furthermore, a multitude of adjectives have been used to describe characteristics of simulation fidelity. These include physical, functional, psychological, behavioural, engineering, visual and auditory, to name but a few (Gerathewohl, 1969; Rehmann et al., 1995). Many of these terms have been adopted from other disciplines such as aviation and engineering into the healthcare simulation literature. This has lead to some misconceptions, whereby fidelity seems to be confused with the level of technological sophistication instead of the "verisimilitude" of an experience, how the tool is actually used and hence how the simulation appears to be true to learners.

In this article, we aim to clarify the term simulation fidelity. First we set out what we mean by simulation and how we distinguish this from the term simulator. We then draw on some current accepted definitions of simulation fidelity as a basis for questioning and clarifying the term's meaning. We present the concept of absolute fidelity as a basis for elucidating what it takes to create increasing fidelity. We then attempt to tackle some of the issues and misconceptions about simulation fidelity in the current literature, in particular the lack of a common standard for determining simulation fidelity in healthcare. Finally, we present a 3-dimensional framework as an approach to conceptualising and positioning healthcare simulation activities to aid research, design, and delivery. 


\section{What do we mean by Simulation Fidelity?}

Before considering simulation fidelity, we will first propose definitions for simulation and simulator, as they are often incorrectly used interchangeably in the literature, which can be problematic.

In this article, we use the definitions provided by Dieckmann and Rall (2007) who defined simulators as the medium which allows users to conduct simulations. Examples of simulators include part-task trainers, mannequins or patient simulators, simulated patients (SP), screen-based environments, and simulated equipment and healthcare environments (Alinier, 2007; Crooltall et al, 1987). Simulators do not necessarily need to be physical - they may take the form of software or even the mind of learners engaging in imaginary activities such as facilitated mental simulations. Simulation is an activity, which represents real or potentially real world activities, including hypothetical situations such as major disasters. Examples can range from the focused practice of a skill such as a surgical procedure to a communication exercise with a simulated patient or a confederate who is someone acting the role of a patient relative or a clinician for a specific purpose in relation to the scenario learning objectives (Sanko et al., 2013). The focus of this article is the fidelity of the simulation experience from the perspective of the learners and not the simulators used.

To begin our discussion of fidelity, we selected three exemplar definitions, which are complementary and reflect the general understanding of the terminology as it is used in the current literature. Feinstein \& Cannon (2002) define fidelity as "the level of realism of a simulation presented to the learner" (p.426). For Alessi (2000), fidelity is "the degree to which a simulation replicates reality" (p.203). Hays \& Singer (1989) define fidelity as "the degree of similarity between the training situation and the operational situation which is simulated." (p.50). From these definitions we can deduce that fidelity is related to "similarity" and "realism" of a simulation, and is a continuum of varying "degrees" or "levels". However, it also raises questions about what realism means in simulation for the purpose of a learning experience and the dependence of fidelity requirements on training objectives (AGARD, 1980; Alinier, 2007; Gerathewohl, 1969).

Feinstein and Cannon's definition (2002), which suggested that fidelity is simply related to the perceived realism to the learner, is problematic, as perceived realism may differ amongst individuals (Reis \& Judd, 2000). A simulation may seem realistic to a novice, because of lack of experience, but appear unrealistic to a more experienced clinician who is better able to detect inaccuracies. Simulation fidelity is therefore dependant not only on user perception, but also accuracy of representation in relation to the real world, such as in terms of the laws of physiology.

Some authors (Alessi, 2000; Hays \& Singer, 1989) suggested that fidelity requires similarity and replication of the real world, but does this mean the objective 
replication of reality atom for atom, element for element? On review of the various types of fidelity described in the literature, a spectrum of definitions ranging from those that are more weighted on an objective, positivistic approach to fidelity (physical, engineering, objective) and those that have more emphasis on subjectivity (psychological, perceptual) appears to exist.

Both approaches can however be problematic. Looking at the subjective end of the spectrum, when determining the realism or fidelity of a simulation of a clinical scenario, would one draw on the opinion of a novice trainee with little experience of a real-life equivalent? The simulated scenario may seem highly realistic to the novice due to their limited understanding, but is in fact grossly inaccurate. High-fidelity must therefore not only take into account user perception, but also in some way be representative of the real clinical situation (Dieckmann et al., 2007a; 2007b), such as in terms of the laws of physiology, anatomy, and social or professional interactions. Horcik et al (2014) referred to the engagement or suspension of disbelief and the relation of the balance between the participants' concern about how close to the simulated work or close to the targeted work a simulation-based experience is. A contributing phase to this "by-in" on the part of the participants resides in the introduction and briefing of the simulation experience to expose the potential limitations of the setting, environment, or the "patient" (Alinier, 2011; Dieckmann et al., 2012).

A purely objective approach to fidelity is also problematic. One way to understanding the nature of fidelity is to examine the top end of the fidelity spectrum, which we term in this article as absolute fidelity. It is a concept used in science fiction where simulation is realistic to the point that it cannot be differentiated from reality (Johansson, 2007). It is a concept commonly used in popular science fiction, examples of which include the Holodeck in Star Trek and the Matrix (Jefferson \& Anderson, 2009; Johansson, 2007). Although this may seem farfetched in terms of what the current state of healthcare simulation can achieve, absolute fidelity is sometimes almost achievable.

We may take for instance, an in-situ simulated clinic consultation for biliary colic, using unannounced SP trained to provide a realistic story, i.e. right upper quadrant pain and all the other cues of patient interactions such as emotion and body language. The SP attends the consultation through the clinician's usual workplace, whilst the clinician is unaware that their patient is really an actor, and proceeds to obtain a history and diagnosis just as he or she normally would during a consultation in the usual work setting. The manner and setting in which the clinician can interact with the SP can be accurate to the degree such that even an expert is unable to detect that it is a simulation, in effect creating near absolute fidelity (Rethans et al., 2007).

In this simulation, although some elements of real clinical practice are actually used, e.g. the consultation room, others such as the patient themselves are representations. The SP does not have any actual pathology (i.e. gallstones), but portrays the symptoms accurately through verbal and emotional cues. Absolute 
simulation fidelity is therefore not necessarily achieved through replication of reality atom for atom, but through accurate representations of real world cues and stimuli.

This approach to simulation can be considered a form of deception, a concept which was introduced by Dieckmann et al. (2007b). Deception, which inherently carries some negative connotation, can be very controversial (Truog \& Meyer, 2013) in that it requires hiding some element of truth. In the example above, the clinician was deliberately not informed that it is a simulated case and it is through some mechanism made to believe that the patient had the actual pathology to allow simulation training of near absolute fidelity. This raises some ethical issues which are currently being researched, for example, how far one should go to deceive a trainee and what effect this may have. The deception can also be linked to the technological aspect of the simulation experience, whereby participants are made to believe that a drain is connected to the patient whereas it is linked to a reservoir located in a control room where an operator adjusts the flow of blood or urine. Another common area that can be perceived as deceptive from the perspective of the learners is the use of well-trained confederates in a scenario and whose role can significantly enhance the level of realism of a scenario thanks to their acting capabilities, but they can also play an important role as purposeful distractors unknowingly to the learners or be supportive colleagues (Sanko et al., 2013). We would however like to expand on Dieckmann's suggestion and instead suggest a more pragmatic approach which we term benevolent deception, a term that is sometimes used in healthcare whereby deception is used to benefit the deceived, in this case the trainee. This is done so specific events can occur realistically during a scenario, bringing up pre-determined learning objectives enhancing the learning experience of the trainees (Alinier, 2011; Sanko et al., 2013).

In practice of course, most simulation is of sub-absolute fidelity which requires simulation designers to establish a fiction contract with learners whereby they are required to accept certain limitations, and in a sense, be willingly deceived (Rudolph et al., 2007). This is indeed an important consideration in current simulation training practice, whereby participants undergo pre-briefing and orientation to the limitations of the simulation to encourage immersion and minimise the negative effects of the unrealistic elements of a simulation on the learners' performance in a scenario. This also prevents participants from drawing on these limitations of the simulation during the debriefing as excuses for lack of immersion or poor performance. This could be illustrated by the response from a candidate to an opening question such as "How did this situation make you feel?" with "It was just a dummy so it did not feel real to me...". The use of open questions is commonly used during debriefings (Kriz, 2010) and hence has associated risks. This type of comment has the potential to undermine the whole simulation process and negatively impact the debriefing which brings clarifications and closure to the learners with regards to their simulation-based experience. It is a critique related to the fidelity of the experience. It may or may not be well founded but it potentially highlights the lack of total engagement or suspension of disbelief which occurs with some learners. It is potentially related to the learners' lack of assimilation of 
information and of the rules of engagement provided during the pre-briefing about the limitations of the technology or environment.

Another problem with simply taking fidelity as the replication of reality is that humans have limited perceptual and sensory capacity, in terms of vision, hearing, touch, taste, and smell (Heißing \& Ersoy, 2010). Human beings are limited in terms of how much of the world around them they can perceive at any point in time. In the case of vision, for instance, we cannot perceive images outside the visible wavelength, such as ultraviolet. Experimental psychology has also shown that human visual perception is limited to 15 million variable pixels per eye beyond which we cannot detect any further detail (Deering, 1998). An established body of research in computer and imaging science, which draws on this understanding of human perceptual limits exists and can inform us how to create realistic representations (Deering, 1998). To put this into the context, consider when creating an image for a virtual simulated laparoscopic procedure - producing an image beyond the resolution detectable by the human eye does not increase fidelity as the user cannot detect details beyond this. Likewise, when creating any simulation, replicating the elements of reality that are beyond our ability to sense and perceive, does not increase the fidelity of a simulation as they do not provide additional cues (Baudisch et al., 2003). Fidelity is therefore quite different from simply replicating every element of reality and requires an understanding of human perception and where benevolent deception or "make believe" can be introduced. Nor can fidelity be judged purely on an individual's perception on how realistic a simulation is as it might be ill-informed or subjective.

In summary, fidelity is an intrinsic property of simulation and can be defined as the degree of accuracy to which a simulation, whether it is physical, mental, or both, represents a given frame of reality in terms of cues and stimuli, and permissible interactions.

\section{Misconceptions and Issues in the Current Literature}

Having clarified the meaning of fidelity, we will now discuss some issues in the current literature. Firstly, as mentioned above, some terminological misconceptions appears to exists, confusing technological sophistication with fidelity (Issenberg et al., 2005; Maran \& Glavin, 2003). A common incorrect assumption we have observed in the literature is that in order to achieve higher levels of fidelity, more advanced (and therefore more expensive) technology is required. For example, simulations using current full-body patient simulators have typically been classified according to their technological specifications, where high-fidelity requires automated computer-controlled model-driven mannequins, whilst intermediate fidelity simulators requires instructor-controlled mannequins (Alinier, 2007; Maran \& Glavin, 2003). Consider a simulation depicting blood pressure dropping in a patient in a state of hypovolaemic shock. Fidelity should be equivalent regardless of whether it is instructor or model-driven, as long as the representation of blood pressure is accurate and changes are in line with the laws of physiology. More advanced 
technology - in this case computer-driven physiological modelling - does not necessarily imply higher fidelity from the perspective of the scenario participants.

Second, many papers describe simulations as high-fidelity, simply because they use a so-called, high-fidelity simulator. Whilst higher fidelity simulators can allow higher fidelity simulations related to specific features they have, a simulation is not necessarily high-fidelity simply because it uses high-fidelity simulators and vice versa. Consider a simulation of clinic consultation using an SP, which is arguably the highest fidelity patient simulator. If this SP is instructed to provide a story in an artificially linear way, perhaps to aid the learning process, the simulation itself cannot be said to be high-fidelity.

Another issue is the current labelling of simulation fidelity, which is loosely and inconsistently labelled as high, intermediate, or low (Ker \& Bradley, 2010). What one describes as high-fidelity may not correspond to another. As such conclusions from reviews such as the work from Norman et al. (2012) comparing learning from high-fidelity simulation versus low-fidelity may not be totally reliable. Low, intermediate, and high-fidelity also do not appear to be equidistant from one another and depends on what element is being characterised. How then can we synthesise the literature to translate research to practice? In addition, how close to absolute fidelity does a simulation have to be to be labelled as high-fidelity? Some so-called high-fidelity simulations are in fact unrealistic in many ways. For example, those using "advanced" interactive patient simulators, which despite being able to provide many physiological cues, do not accurately represent patient interactions, body language, skin colour, and body temperature changes (Dieckmann et al., 2007a; Dieckmann et al., 2007b). Consider what would happen if future developments in simulation allow us to create ever higher levels of fidelity. Would this make these current high-fidelity simulations lower fidelity?

Second, whilst labelling simulations as high, intermediate, and low fidelity allows us to differentiate simulations according to different levels of realism, it is uncertain where to draw the line between them and thus may have limited utility. Low, intermediate, and high-fidelity simulations as they are currently described do not appear to be placed equidistant from one another along the spectrum. How then can educators synthesise the literature to determine fidelity requirements for training and assessment?

This issue is unsurprising given the qualitative nature of these labels. One approach to this problem is to create standardised criteria for each type of simulation. The aviation industry have classified full flight simulation into four levels of fidelity according to increasing realism of cues and stimuli such as motion and physical cockpit design. The highest fidelity simulations use simulators that match specific models of aircraft (Craig, 2003) for example a Boeing 747. This approach however can be problematic for clinical simulations as unlike the fairly standard Boeing 747 , human beings and diseases are infinitely more variable, making them difficult to determine a reference point for labelling fidelity, as we have to recognise that "no one is the average patient" (Alinier, 2007, p.246). 
Nevertheless, we argue that a robust, standardised classification system to provide a unified language for simulation practitioners is needed. In essence, the healthcare simulation community should aim to achieve a consensus on this matter.

In addition, we argue for the need to move away from the unilateral labelling of overall simulation fidelity from low to high, as it provides little useful information in terms of what contributes to the overall fidelity, limiting its utility. Pace (1998) compared this to describing patients as having good or poor health. What clinicians need are the specifics of a patient's condition .This is a recognised issue whereby a number of authors have described simulation fidelity along different dimensions (Beaubien \& Baker, 2004; Pace, 1998). For example, Rehmann et al. (1995) described a typology of flight simulation fidelity currently used in healthcare simulation as follows:

"Equipment (fidelity) cues provide a duplication of the appearance and feel of the operational equipment (the aircraft), i.e., the static and internal dynamic characteristics such as the size, shape, location, and colour of controls and displays, including controller force and displacement characteristics."

"Environment (fidelity) cues provide a duplication of environment and motion through the environment..."

"Perceptual fidelity provides a psychological/physiological standard and is the degree to which the flight crew subjectively perceives the simulator to reproduce its real-life counterpart aircraft, in flight, in the operational task situation."

Similar dimensions of fidelity such as physical and engineering fidelity have also been described by other authors including Miller (1954), Hays and Singer (1989), and Kinkade and Wheaton (1972). Whilst these dimensions provide better descriptions of fidelity, they appear to be better suited for the simulation of machines than for clinical situations. Healthcare simulation has been described according to engineering fidelity, when perhaps it should be according to anatomy and physiology (Issenberg \& Scalese, 2007; Marran \& Glavin, 2003). Crucially, none of these dimensions directly address the representation of patients.

\section{A Three-Dimensional Framework of Simulation Fidelity for Healthcare Education}

To address the issues discussed so far, we propose an alternative, more clinically orientated framework for positioning simulation activities according to three dimensions: the patient, healthcare facility or environment, and clinical scenario (Figure 1), whilst also allowing for the notion of "deception". The arrows starting from the centre of the triangle are used to represent the various possible levels of fidelity (high, intermediate/medium, or low) in each of the three dimensions (Table 1). 


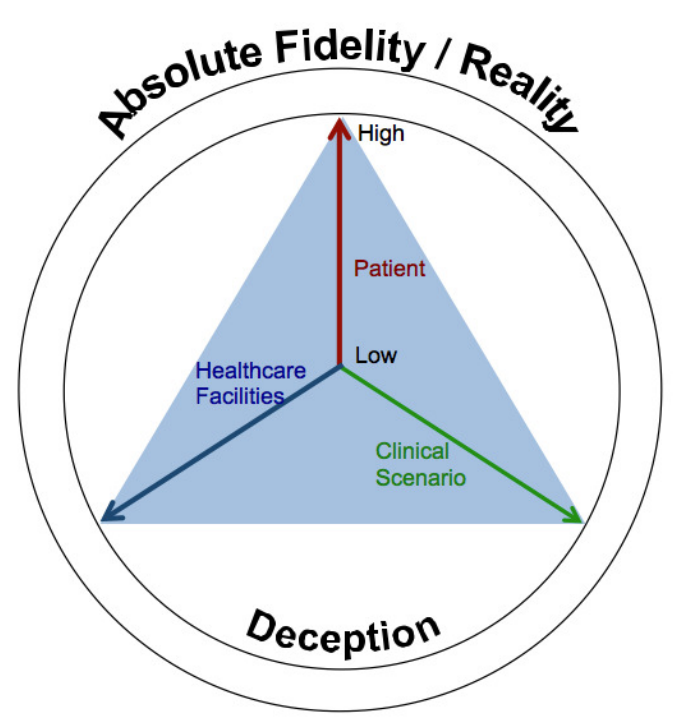

Figure 1: Model of simulation fidelity along the dimensions of Patient, Healthcare Facilities, and Clinical Scenario.

The patient dimension encompasses representations of interactions with all or part of a patient, such as communicating or performing a procedure, and takes into account fidelity of anatomy and physiology. In specific cases, where no patient is involved, this dimension may instead refer to a confederate acting as a patient's relative or colleague with whom the learner needs to interact and discuss an issue as part of simulation-based learning experience. The clinical scenario dimension is related to representations relating to the script and progression of a scenario, and situational complexity such as team and family dynamics. It includes the educators' involvement, whose role is to facilitate the orientation to the experiential learning process and the debriefing that follows it. Interference from an educator during the scenario experience other than in a relevant acting capacity reduces the overall level of simulation fidelity. The healthcare facilities dimension encompasses representations of the clinical equipment and environment, such as the instruments, the monitors, and the environment in which clinical activities or patient encounters take place (Table 1).

Figure 1 shows a framework representing the three dimensions where the level of fidelity along each dimension is increased from minimum to maximum as they project outwards from the centre. Note that in this model, the dimensions are not mutually exclusive and may overlap depending on the type of simulation. For example, in a simulated handover situation a computer screen with patient details can represent both patient and healthcare facilities. This framework however, aims to demonstrate their synergistic effect such that when all three dimensions are at their maximum, absolute fidelity is achieved even if it sometimes involves a degree of deception, which has been represented as an inner circle (Figure 1).

Using this framework, we can proceed to map and describe healthcare simulation activities according to the type of simulation and the characteristics of fidelity. Figure 2 shows an example of how three different simulations ( $A, B$, and $C)$ of venepuncture can be mapped according to the fidelity dimensions, using a real 
clinical encounter of venepuncture on a patient in a clinic for pre-operative blood checks as a frame of reference.
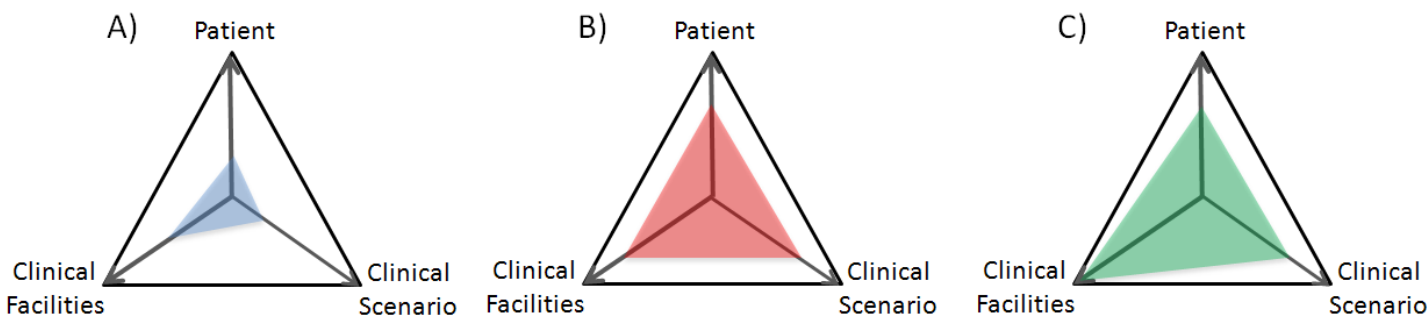

A) Venepuncture simulation using venepuncture arm part-task-trainer in isolation in a skills laboratory, with real clinical equipment (needle, syringe, sample bottles).

B) Patient focused hybrid simulation of venepuncture in skills laboratory, with real clinical equipment (needle, syringe, sample bottles), using a simulated patient wearing a venipuncture pad, framed within a realistic clinical scenario.

C) Patient focused hybrid simulation of venepuncture conducted in-situ (real clinical workplace), with real clinical equipment (needle, syringe, sample bottles), using a simulated patient wearing a venipuncture pad, framed within a realistic clinical scenario.

Figure 2: Example of levels of fidelity of different venepuncture simulation activities along the dimensions of Patient, Healthcare Facilities, and Clinical Scenario.

Along the patient dimension, simulations $B$ and $C$ are accurate representations and higher fidelity in comparison to simulation $A$, through use of hybrid simulation (SPs and venepuncture pad, B and C) as opposed to a venepuncture mannequin in isolation (A). Along the healthcare facilities dimension, fidelity is increased through more realistic representations of the equipment used and the environment in which the simulated venepuncture is conducted, from $A$ (skills laboratory) to B (simulated healthcare environment), and C (in situ, i.e. real healthcare environment). Finally, along the clinical scenario dimension, fidelity is increased from simulation $A$ which is purely task training, to $B$ and $C$ where the simulation is framed within the scenario of unsupervised pre-operative blood checks. Table 1 has been designed to assist in making a more objective appreciation of the levels of fidelity of each dimension of the framework. 


\begin{tabular}{|c|c|c|c|}
\hline Level of Fidelity & Patient & Healthcare facilities & Clinical Scenario \\
\hline Low & $\begin{array}{l}\text { Suboptimal for the } \\
\text { scenario. } \\
\text { Limited anatomical or } \\
\text { physiological } \\
\text { representation of } \\
\text { reality from any } \\
\text { sensory aspect. }\end{array}$ & $\begin{array}{l}\text { Not contextualised to } \\
\text { the scenario. } \\
\text { Element(s) of the } \\
\text { environment need to } \\
\text { be assumed present } \\
\text { by participants. }\end{array}$ & $\begin{array}{l}\text { Task training or } \\
\text { supervised practice. } \\
\text { Constant prompting } \\
\text { by educator(s). } \\
\text { Participants have been } \\
\text { informed of all steps } \\
\text { of the scenario. }\end{array}$ \\
\hline Medium/Intermediate & $\begin{array}{l}\text { Correct anatomical or } \\
\text { physiological } \\
\text { representation in } \\
\text { relation to the } \\
\text { scenario requirements } \\
\text { but presenting some } \\
\text { limitations. }\end{array}$ & $\begin{array}{l}\text { Simulated } \\
\text { environment (i.e. skills } \\
\text { laboratory). } \\
\text { Environment not fully } \\
\text { matching the context } \\
\text { required by the } \\
\text { scenario in terms of } \\
\text { space and equipment } \\
\text { available. }\end{array}$ & $\begin{array}{l}\text { Participant re-enacting } \\
\text { a scenario following a } \\
\text { demonstration of the } \\
\text { same scenario. } \\
\text { Some interruptions by } \\
\text { the educator(s) } \\
\text { Use of a patient } \\
\text { simulator or simulated } \\
\text { patient on which all } \\
\text { interventions required } \\
\text { by the scenario cannot } \\
\text { be fully performed to } \\
\text { demonstrate learning } \\
\text { outcomes. }\end{array}$ \\
\hline High & $\begin{array}{l}\text { Simulated patient } \\
\text { (actor) fully briefed. } \\
\text { Patient simulator with } \\
\text { all features required } \\
\text { for the scenario } \\
\text { allowing participants } \\
\text { to perform } \\
\text { interventions and } \\
\text { experience them as if } \\
\text { it was with a real } \\
\text { patient. }\end{array}$ & $\begin{array}{l}\text { In-situ (Clinical area) } \\
\text { environment matching } \\
\text { the needs of the } \\
\text { scenario. }\end{array}$ & $\begin{array}{l}\text { Autonomous } \\
\text { involvement of } \\
\text { participants following } \\
\text { adequate orientation } \\
\text { and briefing regarding } \\
\text { the equipment, the } \\
\text { environment, and the } \\
\text { expectations in terms } \\
\text { of scenario } \\
\text { participation. } \\
\text { All information } \\
\text { participants are } \\
\text { expected to find about } \\
\text { the patient in the } \\
\text { scenario is available as } \\
\text { per scenario } \\
\text { objectives. }\end{array}$ \\
\hline
\end{tabular}

Table 1: Guidance on determining the level of fidelity for the different dimensions of the proposed framework.

Whilst, we have used venepuncture simulation as an example to explain our framework, we envisage this framework to be applicable to other healthcare simulations which may be simpler as well as more complex whereby they can involve a multiprofessional team of participants. Using this framework, we can set standardised fidelity criteria and be more rigorous in describing our work in the scientific literature, thereby allowing us to make better comparisons between 
simulations of different fidelity levels to determine the true relationship to educational effectiveness. It may also provide a useful platform for guiding simulation design (Scerbo et al., 2011).

\section{Conclusion}

In this article, we have attempted to clarify the meaning of fidelity specifically arguing that simulation fidelity does not necessarily requires faithful replication of reality, but the accurate representation through cues and stimuli from the perspective of the learner or participant. An important aspect of simulation-based training activities for participants is their involvement and the early clarification of limitations to allow for suspension of disbelief. We have also highlighted some terminological issues in the current literature which can make it difficult to translate research to practice and to objectively compare learning outcomes in relation to the simulation fidelity used.

In recognition of the current limitations of describing fidelity, we have proposed an alternative multi-dimensional framework along the axes of the patient, clinical scenario, and healthcare facilities as a means for more precise and practical positioning of healthcare simulations. To clarify the application of the framework, some examples have been presented and discussed. This proposed framework, however, represents just one way of considering fidelity of healthcare simulation. We hope that this article will be a catalyst for further debate and scholarship on this difficult but very necessary topic.

\section{Acknowledgement}

The authors wish to acknowledge the contribution of Dr Peter Dieckmann as an active participant during the presentation of this framework at a workshop of the 2011 meeting of the Society in Europe for Simulation Applied to Medicine (SESAM) when he emphasised the notion of "Deception" to help achieve the desire level of fidelity. Thank you also to the anonymous reviewers for the critic of the article and the valuable suggestions made by Dr Tim Clapper. Finally, we would like to thank the Simulation and Technology enhanced Learning Initiative (STeLi) group at the London Deanery, UK, for their support in our research in simulation education in healthcare.

\section{Author contributions}

All authors contributed to this article, in content and in form. Initial concept by JKT. All authors worked on the conceptual development. JKT, GA, and RLK contributed to the SESAM workshop. JKT, GA, JT, and RLK wrote the manuscript. JKT and GA designed the graphics. 


\section{$\underline{\text { References }}$}

AGARD (1980). Fidelity of simulation for pilot training. Advisory group for aerospace research and development Neuilly-sur-Seine, December 1980.

Aggarwal, R., O. T. Mytton, M. Derbrew, D. Hananel, M. Heydenburg, B. Issenberg, C. MacAulay, et al. (2010). Training and simulation for patient safety. Quality and Safety in Health Care, 19, no. Suppl 2, i34-i43.

Alessi, S. (2002). Building versus using simulations. In: Spector JM, Anderson TM, eds. Integrated and Holistic Perspectives on Learning, Instruction and Technology. Dordrecht: Kluwer Academic Publishers, 175-196.

Alessi, S. (2000). Simulation design for training and assessment. In: Aircrew training and assessment. Routledge. 197-222.

Alinier, G., Platt, A. (2014). International overview of high-level simulation education initiatives in relation to critical care. Nursing in Critical Care, 19(1), 42-49.

Alinier, G. (2007). A typology of educationally focused medical simulation tools. Medical Teacher, 29(8), e243-250.

Alinier, G. (2011). Developing high-fidelity health care simulation scenarios: A guide for educators and professionals. Simulation \& Gaming, 42(1):9-26.

Baudisch, P., DeCarlo, D., Duchowski, A.T., Geisler, W.S. (2003). Focusing on the essential: considering attention in display design. Communications of the ACM, 46(3),60-66.

Beaubien, J.M., Baker, D.P. (2004). The use of simulation for training teamwork skills in health care: how low can you go? Quality and Safety in Health Care, 13(suppl_1), i51i56.

Borodzicz, E. (2004). The missing ingredient is the value of flexibility. Simulation \& Gaming, 35, 414-426.

Bredmose P.P., Habig K., Davies G., Grier G., Lockey D.J. (2010). Scenario based outdoor simulation in pre-hospital trauma care using a simple mannequin model. Scan J Trauma Resusc Emerg Med, 18(13), 1-6

Craig, P.A. (2003). Multi-Engine Flying. McGraw-Hill Professional.

Chiniara, G., Cole, G., Brisbin, K., Huffman, D., Cragg, B., Lamacchia, M., \& Norman, D. (2013). Simulation in healthcare: A taxonomy and a conceptual framework for instructional design and media selection. Medical Teacher, 35, e1380-e1395.

Crooltall, D., Oxford, R., \& Saunders, D. (1987). Towards a Reconceptualization of Simulation: From Representation to Reality. Simulation/Games for Learning, 17(4), 147-71.

Deering, M.F. (1998). The limits of human vision. In: 2nd International Immersive Projection Technology Workshop, 2.

Dieckmann, P., Rall, M. (2007). Simulators in anaesthetic training to enhance patient safety. In: Recent advances in anaesthesia and intensive care, 24. Recent Advances. Cambridge University Press, 211-232

Dieckmann, P., Gaba, D., Rall, M. (2007a). Deepening the theoretical foundations of patient simulation as social practice. Simulation in Healthcare, 2(3), 183-193.

Dieckmann, P., Manser, T., Wehner, T., Rall, M. (2007b). Reality and fiction cues in medical patient simulation: An interview study with anesthesiologists. Journal of Cognitive Engineering and Decision Making, 1(2), 148-168. 
Dieckmann, P., Friis, S. M., Lippert, A., \& Østergaard, D. (2012). Goals, success factors, and barriers for simulation-based learning: A qualitative interview study in health care. Simulation \& Gaming, 43(5), 627-647.

Feinstein, A.H., Cannon, H.M. (2002). Constructs of simulation evaluation. Simulation \& Gaming, 33(4), $425-440$.

Gaba, D.M. (2004). The future vision of simulation in health care. Quality and Safety in Health Care, 13(suppl_1), i2-i10.

Gerathewohl, S. J. (1969). Fidelity of simulation and transfer of training: A review of the problem. Report AM 69-24. Department of transportation, Federal Aviation Administration, Office of Aviation Medicine, USA

Hays, R.T., Singer, M.J. (1989). Simulation fidelity in training system design: bridging the gap between reality and training. Springer-Verlag.

Heißing, B., Ersoy, M. (2010). Chassis Handbook: Fundamentals, driving dynamics, components, mechatronics, perspectives. Vieweg+Teubner Verlag.

Horcik, Z., Savoldelli, G., Poizat, G., \& Durand, M. (2014). A phenomenological approach to novice nurse anesthetists' experience during simulation-based training sessions. Simulation in Healthcare, 9(2), 94-101

Issenberg, S.B., Mcgaghie, W.C., Petrusa, E.R., Lee Gordon, D., Scalese, R.J. (2005). Features and uses of high-fidelity medical simulations that lead to effective learning: a BEME systematic review. Medical Teacher, 27(1), 10-28.

Issenberg, S.B., Scalese, R.J. (2007). Simulation in health care education. Perspectives in Biology and Medicine, 51(1), 31-46.

Jefferson, M., Anderson, M. (2009). Enter the matrix: the relationship between drama and film. In: Drama Education with Digital Technology. Continuum International Publishing Group, 184-202.

Johansson, M. (2007). When simulations become reality. National Course in Philosophy of Computer Science. Available at: http://www.idt.mdh.se/personal/gdc/work/PedagogogicCareerLadder/Appendices/ Appendix-C/C2-PI-CourseProceedings.pdf\#page=79 Accessed July 4, 2014.

Kardong-Edgren, S.E., Anderson, M., \& Michaels, J. (2007). Does simulation fidelity improve student test scores? Clinical Simulation in Nursing Education, 3, e21-e24.

Ker, J., Bradley, P. (2010). Simulation in medical education. In: Understanding medical education: Evidence, theory and practice. John Wiley and Sons, 164-180.

Kinkade, R.G., \& Wheaton, G.R. (1972). Training device design. Human engineering guide to equipment design. Department of Defence Washington, 668-699.

Kneebone, R., Aggarwal, R. (2009). Surgical training using simulation. BMJ, 338, b1001.

Kriz, W. C. (2010). A systemic-constructivist approach to the facilitation and debriefing of simulations and games. Simulation \& Gaming, 41(5), 663-680.

Lane, N.E., Alluisi, E.A. (1992). Fidelity and Validity in Distributed Interactive Simulation: Questions and Answers. Available at: www.dtic.mil/dtic/tr/fulltext/u2/a260971.pdf. Accessed July 4, 2014.

Lapkin, S., \& Levett-Jones, T. (2011). A cost-utility analysis of medium vs. high-fidelity human patient simulation manikins in nursing education. Journal of Clinical Nursing, 20, 3543-3552.

Lee, K.H.K., Grantham, H., Boyd, R. (2008). Comparison of high- and low-fidelity mannequins for clinical performance assessment. Emergency Medicine Australasia, 20(6), 508514. 
Levett-Jones, T., Lapkin, S., Hoffman, K., Arthur, C., \& Roche, J. (2011). Examining the impact of high and medium fidelity simulation experiences on nursing students' knowledge acquisition. Nurse Education in Practice, 11(6), 380-383.

Liu, A., Tendick, F., Cleary, K., Kaufmann, C. (2003). A survey of surgical simulation: applications, technology, and education. Presence: Teleoperators \& virtual environments, 12(6), 599-614.

Maran, N.J., Glavin, R.J. (2003). Low- to high-fidelity simulation - a continuum of medical education? Med Educ, 37, Suppl 1,22-28.

Mcgaghie, W.C., Issenberg, S.B., Petrusa, E.R., Scalese, R.J. (2010). A critical review of simulation-based medical education research: 2003-2009. Med Educ, 44(1),50-63.

Meakim, C., Boese, T., Decker, S., Franklin, A.E., Gloe, D., Lioce, L., Sando, C.R., \& Borum, .JC. (2013). Standards of best practice: Simulation Standard I: Terminology. Clinical Simulation in Nursing, 9(6), S3-S11.

Miller, R.B. (1954). Psychological considerations in the design of training equipment. DTIC document. Available at:

http://oai.dtic.mil/oai/oai?verb=getRecord\&metadataPrefix=html\&identifier=AD007 1202. Accessed July 4, 2014.Norman, G., Dore, K., \& Grierson, L. (2012). The minimal relationship between simulation fidelity and transfer of learning. Medical education, 46(7), 636-647.

Pace, D.K. (1998). Dimensions and attributes of simulation fidelity, 1998 Fall Simulation Interoperability Workshop Papers.

Paige, J.B., \& Morin, K.H. (2013). Simulation fidelity and cueing: A systematic review of the literature. Clinical Simulation in Nursing, 9(11), e481-e489.

Rehmann, A.J., Mitman, R.D., Reynolds, M.C. (1995). A handbook of flight simulation fidelity requirements for human factors Research. Available at: http://www.dtic.mil/get-trdoc/pdf?AD=ADA303799. Accessed July 4, 2014.

Reis, H.T., Judd, C.M. (2000). Handbook of research methods in social and personality psychology. Cambridge University Press.

Rethans, J-J., Gorter, S., Bokken, L., Morrison, L. (2007). Unannounced standardised patients in real practice: a systematic literature review. Med Educ. 41(6), 537-549.Rudolph, J.W., Simon, R., Raemer, R. 2007. Which reality matters? Questions on the path to high engagement in healthcare simulation. Simulation in Healthcare, 2(3), 161-163.

Sanko, J. S., Shekhter, I., Kyle Jr, R. R., Di Benedetto, S., \& Birnbach, D. J. (2013). Establishing a convention for acting in healthcare simulation: merging art and science. Simulation in Healthcare, 8(4), 215-220.

Scerbo, M.W., Bosseau Murray, W., Alinier, G., Antonius, T., Caird, J., Stricker, E., Rice, J., Kyle, R. (2011). A path to better healthcare simulation systems: Leveraging the integrated systems design approach. Simulation in Healthcare, 6, S20-S23.

Schijven, M., Jakimowicz, J. (2003). Virtual reality surgical laparoscopic simulators. Surgical endoscopy, 17(12), 1943-1950.

Truog, R., D., \& Meyer, E., C. (2013). Deception and in medical simulation. Simulation in Healthcare, 8(1), 1-3

Ziv, A., Wolpe, P.R., Small, S.D., Glick, S. (2003). Simulation-based medical education: An ethical imperative. Acad Med, 78(8), 783-788. 


\section{Bios}

Jimmy Kyaw Tun, is a Specialist Registrar in Radiology at Bart's Health and a doctorate student and research fellow at Imperial College. He is actively involved in both undergraduate and postgraduate medical education and has previously completed a Masters degree in clinical education at the Institute of Education. His research lies in clinical education, in particular simulation-based education. Contact: jimms@doctors.net.uk

Guillaume Alinier, PhD, SFHEA, is an active member of several Societies and has received multiple awards for his contributions in learning and teaching, primarily in scenario-based simulation education and OSCE assessment. He received his Professorship in 2011 from the University of Hertfordshire and is currently based in Doha, where he supports research and training at Hamad Medical Corporation Ambulance Service. Contact: g.alinier@herts.ac.uk

Jessica Tang, is a Chartered Applied Psychologist and a Fellow of Royal Society for Public Health. She was a post-doctoral research psychologist in the Division of Surgery of Imperial College London and lead researcher of the Distributed Simulation project. Jessica is currently working in the Hong Kong Hospital Authority and responsible for the development and coordination of medical simulation training. Contact: tjj571@ha.org.hk

Roger L. Kneebone, had a clinical career that encompassed trauma surgery and general practice. As an academic, he has an international profile as an innovator, with a special interest in contextualised simulation. He leads a multidisciplinary research team which brings together clinicians, scientists, artists and performers. He is Professor of Surgical Education at Imperial College London and Wellcome Trust Engagement Fellow. Contact: r.kneebone@imperial.ac.uk 